\title{
Open
}

\section{Changes in plasma and urine globotriaosylceramide levels do not predict Fabry disease progression over 1 year of agalsidase alfa}

\author{
Raphael Schiffmann, MD¹, Markus Ries, MD, PhD², Derek Blankenship, $\mathrm{PhD}^{3}$, Kathy Nicholls, $\mathrm{MD}^{4}$, \\ Atul Mehta, $\mathrm{MD}^{5}$, Joe T.R. Clarke, MD, PhD ${ }^{6}$, Robert D. Steiner, $\mathrm{MD}^{7}$, Michael Beck, $\mathrm{MD}^{8}$, \\ Bruce A. Barshop, MD, PhD ${ }^{9}$, William Rhead, MD ${ }^{10}$, Michael West, MD, FRCPC ${ }^{11}$, Rick Martin, MD ${ }^{12}$, \\ David Amato, $\mathrm{PhD}^{13}$, Nitin Nair, $\mathrm{PhD}^{12}$ and Pedro Huertas, MD, PhD ${ }^{14,15}$
}

Purpose: Globotriaosylceramide concentrations were assessed as potential predictors of change from baseline after 12 months by estimated glomerular filtration rate and left-ventricular mass index using pooled data from three randomized, placebo-controlled agalsidase alfa trials and open-label extensions of patients with Fabry disease.

Methods: Males (aged 18 years or older) with Fabry disease received agalsidase alfa $(0.2 \mathrm{mg} / \mathrm{kg}$ every other week for 12 months). A backward-elimination approach evaluated potential predictors (baseline estimated glomerular filtration rate and left-ventricular mass index; age at first dose; baseline and change from baseline at 12 months of globotriaosylceramide (urine, plasma); urine protein excretion; and systolic and diastolic blood pressure). Subgroups included patients randomized to placebo or agalsidase alfa (double-blind phase), then to agalsidase alfa (open-label extensions; placebo $\rightarrow$ agalsidase alfa or agalsidase alfa $\rightarrow$ agalsidase alfa, respectively) and stage $2 / 3$ chronic kidney disease patients.

Globotriaosylceramide $\left(\mathrm{Gb}_{3}\right)$ is often elevated in the urine of patients with Fabry disease, ${ }^{1}$ and some studies support its use as a diagnostic biomarker. ${ }^{2-4}$ Plasma $\mathrm{Gb}_{3}$ concentration has been found to be consistently elevated in hemizygous males with classic Fabry disease but variably elevated in some variant hemizygous males with residual enzyme activity and in heterozygous females. ${ }^{5,6}$ No evidence has been published supporting the use of plasma or urine $\mathrm{Gb}_{3}$ concentrations as a biomarker for disease progression or response to treatment. For patients with elevated plasma or urine $\mathrm{Gb}_{3}$ concentrations before treatment, enzyme replacement therapy (ERT) results in an initial drop in $\mathrm{Gb}_{3}$ concentrations. ${ }^{5,7}$ The lower $\mathrm{Gb}_{3}$ concentrations do not remain low in all patients and do not always coincide with clinical improvement. ${ }^{8}$
Results: Baseline estimated glomerular filtration rate, age at first dose, baseline urine globotriaosylceramide excretion, and baseline and change from baseline urine protein excretion significantly predicted change from baseline estimated glomerular filtration rate in the analysis population $(N=73$; all $P<0.05)$, although not in all subgroups. Change from baseline urine and plasma globotriaosylceramide (baseline and change from baseline) concentrations did not predict change from baseline estimated glomerular filtration rate. No predictors of left-ventricular mass index were significant.

Conclusion: Changes in globotriaosylceramide concentrations do not appear to be useful biomarkers for prediction of Fabry disease-related changes in estimated glomerular filtration rate or leftventricular mass index.

Genet Med advance online publication 16 May 2013

Key Words: agalsidase alfa; biomarkers; enzyme replacement therapy; Fabry disease; globotriaosylceramide

Biomarkers are generally defined as measurements that reflect the activity of a disease process ${ }^{9}$ and can be (i) prognostic, (ii) predictive, or (iii) pharmacodynamic. ${ }^{10}$

The goal of this study was to assess the relationship of plasma and urine $\mathrm{Gb}_{3}$ concentrations with renal or cardiac outcome measures. A previous analysis of pooled data from three randomized, placebo-controlled clinical trials and their open-label extensions (sponsored by Shire Human Genetic Therapies) of male patients with Fabry disease suggested a stabilizing effect of agalsidase alfa (agal $\alpha$ ) on renal function assessed by measured glomerular filtration rate (GFR). ${ }^{11}$ In that analysis, baseline GFR or elevated proteinuria category $(\geq 1 \mathrm{~g} / 24 \mathrm{~h})$ significantly predicted GFR decline during treatment. Using a suitable selection approach of pooled data

\footnotetext{
The first two authors contributed equally to this work.

${ }^{1}$ Institute of Metabolic Disease, Baylor Research Institute, Dallas, Texas, USA; ${ }^{2}$ Pediatric Neurology, Center for Pediatric and Youth Medicine, University Clinic Heidelberg, Heidelberg, Germany; ${ }^{3}$ Department of Quantitative Sciences, Baylor Institute for Health Care Research and Improvement, Dallas, Texas, USA; ${ }^{4}$ Department of Nephrology, Royal Melbourne Hospital and University of Melbourne, Parkville, Australia; ${ }^{5}$ Lysosomal Storage Disorders Unit, Department of Haematology, Royal Free Hospital, London, UK; ${ }^{6}$ Division of Clinical and Metabolic Genetics, Hospital for Sick Children and University of Toronto, Toronto, Ontario, Canada; ${ }^{7}$ Department of Pediatrics and Molecular and Medical Genetics, Oregon Health \& Science University, Portland, Oregon, USA; ${ }^{8}$ Centre for Lysosomal Storage Disorders, University of Mainz, Mainz, Germany; ${ }^{9}$ Metabolic Genetics, Rady Children's

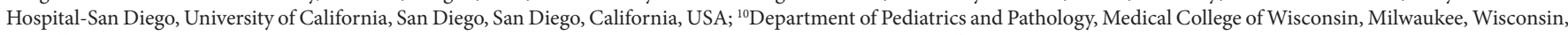
USA; ${ }^{11}$ Division of Nephrology, Department of Medicine, Dalhousie University, Halifax, Nova Scotia, Canada; ${ }^{12}$ Shire Human Genetic Therapies, Lexington, Massachusetts, USA; ${ }^{13}$ Vertex Pharmaceuticals, Cambridge, Massachusetts, USA; ${ }^{14}$ Medicines Development Group, Neurosciences, Pfizer Inc, Cambridge, Massachusetts, USA; ${ }^{15}$ Massachusetts General Hospital and Division of Health Sciences and Technology, Harvard Medical School-Massachusetts Institute of Technology, Boston, Massachusetts, USA. Correspondence: Raphael Schiffmann (Raphael.Schiffmann@baylorhealth.edu)
} 
from the same three clinical trials, we asked whether $\mathrm{Gb}_{3}$ concentrations could also be a predictor of changes in kidney function and/or left-ventricular mass index (LVMI) in a large study population of patients with Fabry disease receiving agal $\alpha$ ERT.

\section{MATERIALS AND METHODS \\ Clinical trial designs and treatments}

Data were pooled from three 24-week, randomized, doubleblind, placebo-controlled trials (RCTs) and their open-label extension studies (EXTs; TKT003/TKT006; TKT005/TKT007; TKT010/TKT013; and TKT015). Two of these trials (TKT003 and extension ${ }^{12}$ and TKT005 and extension) ${ }^{13}$ were singlecenter phase II trials, and one was a multicenter phase III trial (TKT010 and extension).

Treatments in these trials included agal $\alpha(0.2 \mathrm{mg} / \mathrm{kg}$ body weight) infused intravenously over a 40 -min period every other week or placebo (infused on the same schedule). For these analyses, patient data were analyzed for 12 months of total agal $\alpha$ treatment (Figure 1). Patients were either treated with agal $\alpha$ for 6 months (RCTs), followed by an additional 6 months of agal $\alpha$ (EXTs), or they received placebo for 6 months (RCTs) and then transitioned to 12 months of agal $\alpha$ (EXTs).

\section{Patient selection}

Patients were adult males (aged 18 years or older) with Fabry disease (OMIM 301500) confirmed by clinical characteristics and alpha-galactosidase A deficiency, who were otherwise considered to have adequate general health. Each individual RCT also had specific inclusion criteria, including symptoms of neuropathic pain (TKT003, TKT010), medication for neuropathic pain at screening (TKT010), and left-ventricular hypertrophy
(TKT005). None of these trials had specific renal inclusion criteria.

For inclusion in these post hoc analyses, patients in the renal analysis population were required to have available data on estimated GFR (eGFR), ${ }^{14}$ plasma $\mathrm{Gb}_{3}$ concentration, urine $\mathrm{Gb}_{3}$ excretion, urine protein excretion, systolic and diastolic blood pressure, and age at baseline and 12 months of agal $\alpha$ treatment. The cardiac analysis population must have had cardiac magnetic resonance imaging measurements for LVMI. All patients should have received at least one dose of agal $\alpha$ during the treatment period assessed. In addition, subpopulations were evaluated comprising patients who were initially randomized to the placebo group in the 6-month RCTs and transitioned to 12 months of agal $\alpha$ in the EXTs (placebo $\rightarrow$ agal $\alpha$; Figure 1), who were initially randomized to agal $\alpha$ (6-month RCTs) and continued on to 6 months of agal $\alpha$ during the EXTs (agal $\alpha \rightarrow$ agal $\alpha$; Figure 1), or who had stage $2 / 3$ chronic kidney disease (CKD 2/3) at baseline (defined as patients with baseline eGFR of $30-90 \mathrm{ml} / \mathrm{min} / 1.73 \mathrm{~m}^{2}$ ).

All studies included in these analyses were approved by the appropriate institutional review boards of the investigators' institutions, and all patients provided written informed consent.

\section{Measurement of eGFR and LVMI}

These analyses use eGFR instead of measured GFR because eGFR allows for the inclusion of more patients in the analysis population. eGFR $\left(\mathrm{ml} / \mathrm{min} / 1.73 \mathrm{~m}^{2}\right)$ was calculated using the Chronic Kidney Disease Epidemiology Collaboration equation, ${ }^{14}$ which incorporates serum creatinine ( $\mathrm{SCr}[\mathrm{mg} / \mathrm{dl}]$ ), the patient's age, sex, and race. $\kappa$ Corresponds to a value of 0.7 (females) or 0.9 (males), and $\alpha$ is -0.329 (females) or -0.411 (males). The "min" value indicates the minimum of SCr/ $\mathrm{k}$ or 1 ,

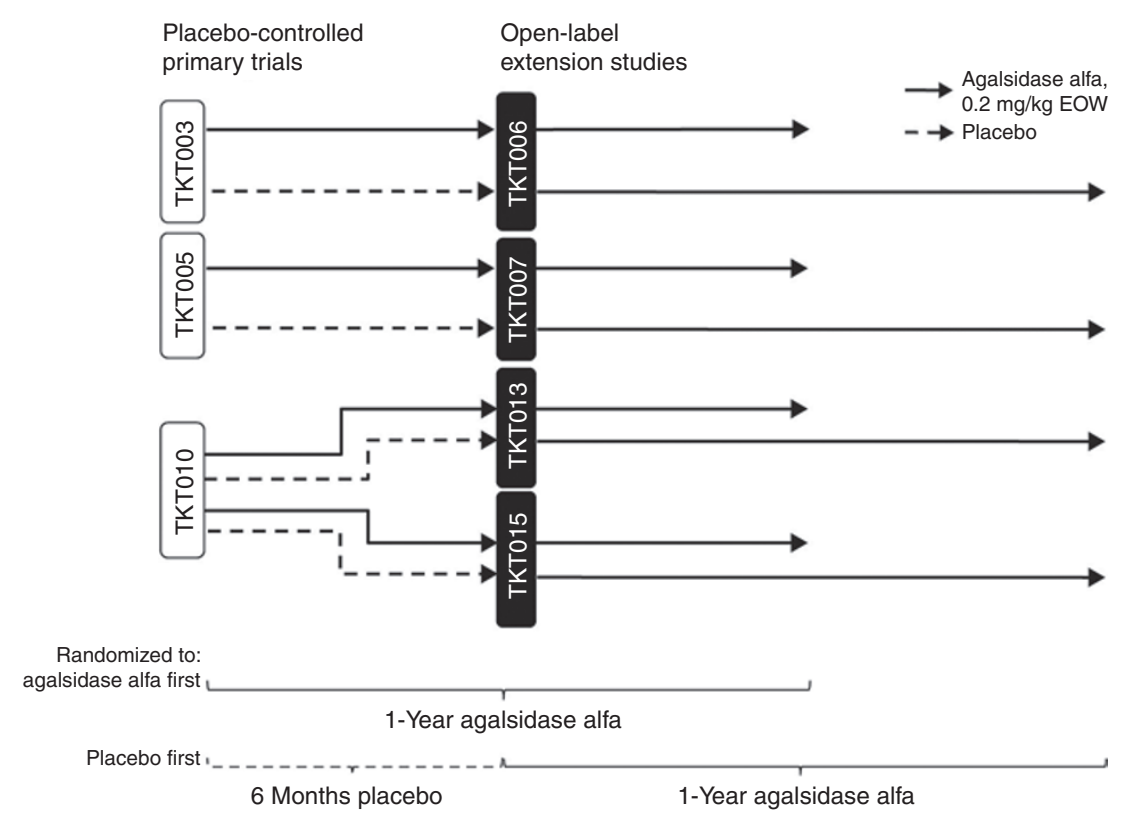

Figure 1 Clinical trial designs and treatment durations for these analyses. Each patient in these analyses received 12 total months of agalsidase alfa treatment (solid lines): either 6 months agalsidase alfa in the double-blind primary trial plus 6 more months in the open-label extension study or placebo in the primary trial (dashed line) plus 12 months of agalsidase alfa in the extension. EOW, every other week. 
and "max" is the maximum of SCr/ $\kappa$ or 1 . The Chronic Kidney Disease Epidemiology Collaboration formula can be expressed as a single equation as follows:

$$
\begin{aligned}
\mathrm{eGFR}=14 & \times \min (\mathrm{SCr} / \kappa, 1)^{\alpha} \times \max (\mathrm{SCr} / \kappa, 1)^{-1.209} \\
& \times 0.993^{\mathrm{Age}} \times 1.018(\text { if female }) \times 1.159(\text { if black })
\end{aligned}
$$

SCr was measured in local laboratories as part of the standard assessments. A local clinical laboratory quantitatively determined 24-h urine protein excretion. Renal disease was staged into CKD subgroups..$^{15}$ LVMI was calculated using cardiac magnetic resonance imaging measurements and using techniques described previously. ${ }^{13}$

\section{Measurement of $\mathrm{Gb}_{3}$ concentration}

Plasma samples were collected for assessment of $\mathrm{Gb}_{3}$ after $8 \mathrm{~h}$ of fasting before drawing the blood for analyses. Urine $\mathrm{Gb}_{3}$ excretion was evaluated in 24-h urine sediment. Both plasma and urine samples were analyzed with high-performance liquid chromatography using a validated assay, as described previously. ${ }^{16}$

\section{Potential predictors}

In the renal analysis population, the primary outcome measure was the change from baseline (CFB) value in eGFR at 12 months. The potential predictors considered for renal outcomes included baseline (before agal $\alpha$ treatment) eGFR and age at start of agala, as well as baseline and CFB (at month 12) plasma $\mathrm{Gb}_{3}$ concentrations, urine $\mathrm{Gb}_{3}$ excretion, natural $\log (\ln )$ of urine protein excretion, and systolic and diastolic blood pressure. In the cardiac analysis population, the same potential predictors (except baseline eGFR) as well as baseline LVMI were evaluated for CFB in LVMI $\left(\mathrm{g} / \mathrm{m}^{2.7}\right)$ at month 12. Interaction effects were also evaluated as described below.

\section{Statistical analyses}

The significance of potential predictors of renal outcomes was assessed via a backward-elimination linear regression model, which started with the full model, including interaction terms, and systematically and sequentially factored out all nonsignificant potential explanatory variables one by one, starting with the least significant variable. Nonsignificant $(P>0.05)$ interaction terms were removed initially, followed by the removal of nonsignificant $(P>0.05)$ main effects that were not a component of a statistically significant interaction term. Because the starting model contains interaction terms, the elimination process did not permit the removal of a main effect term before all interactions related to that main effect were removed. The elimination model was terminated when all remaining main effect terms were either significant or part of a significant interaction term $\left(P_{\text {elimination }}>0.05\right)$. The backwardelimination approach was chosen because it selects models that are more explanatory. A similar approach was used for the cardiac outcomes, with the exception of inclusion of interaction terms due to small sample size. The robustness of the model was also assessed using a linear regression analysis in the three patient subpopulations: placebo $\rightarrow$ agal $\alpha$, agal $\alpha \rightarrow$ agal $\alpha$, and CKD 2/3 subgroups. A Student's $t$-test was used to calculate $P$ values, assessing whether parameters (intercepts and slopes) were significantly different from zero. Statistical significance levels were set at $\alpha=0.05$ (two-sided).

\section{RESULTS}

Baseline demographic and clinical characteristics are shown in Table 1. These characteristics were similar among the entire renal analysis population and the placebo $\rightarrow$ agal $\alpha$ and agal $\alpha \rightarrow$ agal $\alpha$ subgroups. Patients in the CKD 2/3 subgroup had lower baseline eGFR than the whole analysis population and other subpopulations, as expected from the criteria for defining CKD 2/3 (baseline eGFR of 30-90 $\mathrm{ml} / \mathrm{min} / 1.73 \mathrm{~m}^{2}$ ).

Baseline and CFB values at month 12 are shown for each of the parameters evaluated as a potential predictor of renal function (Table 2). The backward-elimination approach identified baseline eGFR, age at start of agal $\alpha$, baseline urine $\mathrm{Gb}_{3}$ excretion, log-transformed baseline, and CFB of urine protein excretion at month 12 as significant predictors of renal function in the renal analysis population (all $P<0.05$; Table 3 ). Furthermore, significant interactions were found between baseline eGFR and log-transformed baseline and CFB in urine protein excretion at month 12 (both $P<0.005$; Table 3). No

\begin{tabular}{|c|c|c|c|c|}
\hline Characteristic & $\begin{array}{l}\text { Analysis population } \\
\text { (all subjects), } N=73\end{array}$ & $\begin{array}{l}\text { Pbo } \rightarrow \text { agal } \alpha \text { subgroup } \\
\qquad(n=36)\end{array}$ & $\begin{array}{c}\text { Agal } \alpha \rightarrow \text { agal } \alpha \text { subgroup }^{b} \\
\qquad(n=37)\end{array}$ & $\begin{array}{c}\text { CKD 2/3 subgroup } \\
(n=25)\end{array}$ \\
\hline $\begin{array}{l}\text { Age at first dose, years, median } \\
\text { (range) }\end{array}$ & $36(20-53)$ & $36(20-52)$ & $35(20-53)$ & $40(26-53)$ \\
\hline \multicolumn{5}{|l|}{ Race, $n(\%)$} \\
\hline White & $66(90)$ & $33(92)$ & $33(89)$ & $21(84)$ \\
\hline Other & $7(10)$ & $3(8)$ & $4(11)$ & $4(16)$ \\
\hline eGFR, $\mathrm{ml} / \mathrm{min} / 1.73 \mathrm{~m}^{2}$, mean $\pm \mathrm{SD}$ & $92.6 \pm 32.4$ & $88.9 \pm 37.7$ & $96.2 \pm 26.3$ & $66.6 \pm 16.0$ \\
\hline
\end{tabular}

Table 1 Demographic and clinical characteristics: analysis population and subgroups

Agalo, agalsidase alfa; CKD, chronic kidney disease; eGFR, estimated glomerular filtration rate; EXT, extension study; Pbo, placebo; RCT, randomized placebo-controlled trial.

apbo $\rightarrow$ agal $\alpha$ subgroup: 6 months placebo (RCT), followed by 12 months agal $\alpha$ (EXT). ${ }^{\text {}}$ Agal $\alpha \rightarrow$ agal $\alpha$ subgroup: 6 months agal $\alpha(R C T)$, followed by 6 months agal $\alpha(E X T)$. ${ }^{c} C K D$ 2/3 subgroup: stage 2/3 CKD before beginning agal $\alpha$ treatment. 
Table 2 Potential predictors (baseline and CFB at month 12) of renal function: possible covariates in 73 patients in the renal analysis population

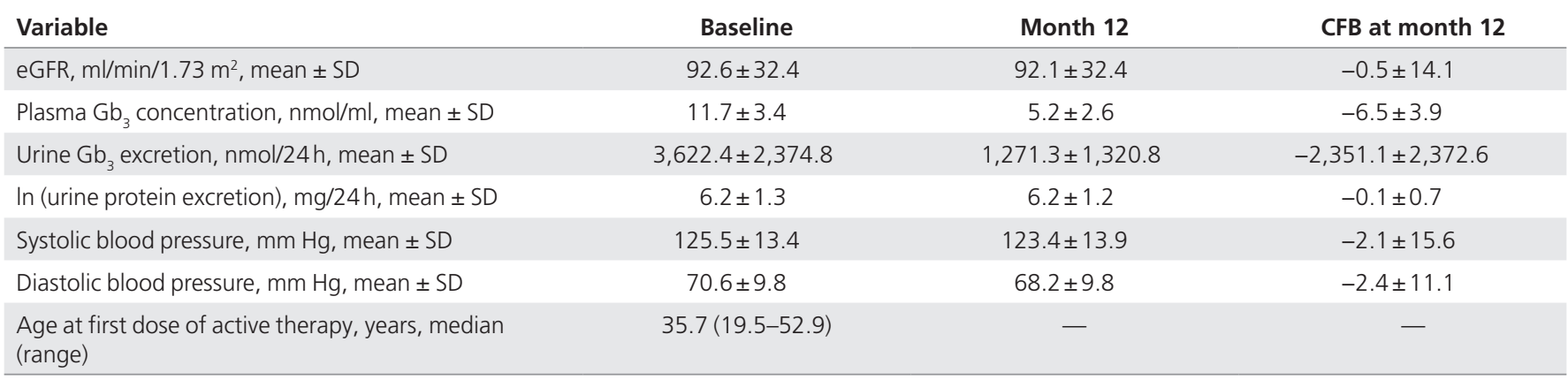

CFB, change from baseline; eGFR, estimated glomerular filtration rate; Gb $3^{\prime}$ globotriaosylceramide.

Table 3 Potential predictors of renal function: backward-elimination approach in 73 patients in the renal analysis population

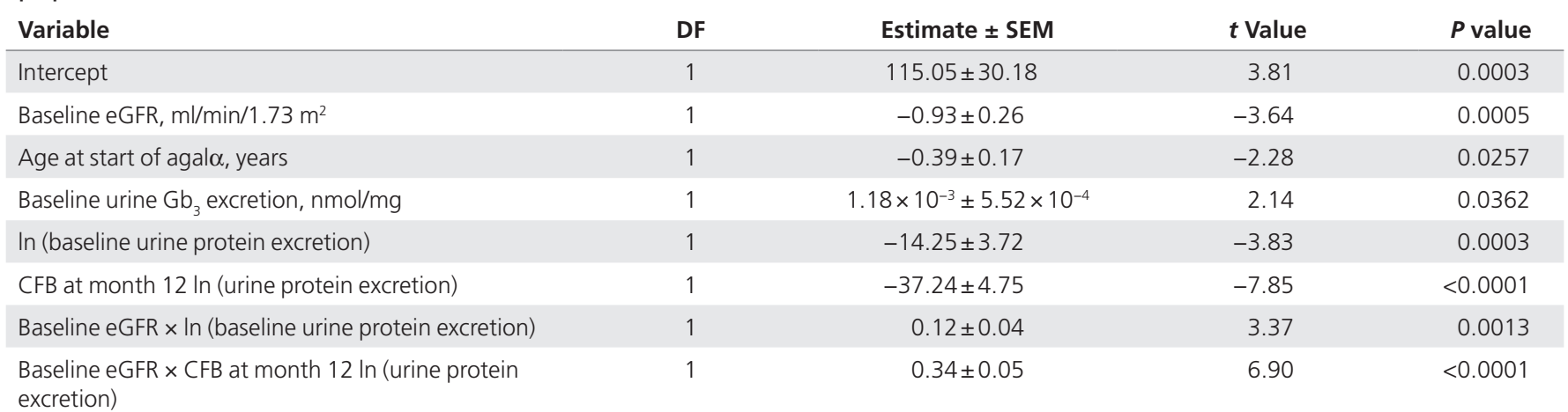

Agal $\alpha$, agalsidase alfa; CFB, change from baseline; DF, degrees of freedom; eGFR, estimated glomerular filtration rate; $\mathrm{Gb}_{3^{\prime}}$ globotriaosylceramide.

other variables assessed were significant predictors of renal function. Most of these potential parameters and interactions were found to be statistically significant predictors of renal function in the placebo $\rightarrow$ agal $\alpha$ subgroup (all $P<0.05$ ), with the exception of age at start of agal $\alpha(P=0.0571$; Table 4$)$. Patients in the agal $\alpha \rightarrow$ agal $\alpha$ subgroup retained only baseline eGFR and log-transformed baseline urine protein excretion as potential predictors (both $P<0.05$ ), with a significant interaction found between these two parameters $(P=0.0153$; Table 4$)$. For CKD $2 / 3$ patients, only urine $\mathrm{Gb}_{3}$ was determined to be a significant predictor of CFB eGFR at month $12(P=0.0105$; Table 4$)$.

The backward-elimination approach did not identify any predictors of CFB LVMI at month 12 (Supplementary Table S1 online).

\section{DISCUSSION}

A large unmet need exists for finding and validating accurate biomarkers in lysosomal storage disorders and, in particular, for Fabry disease. ${ }^{17,18}$ Individual lysosomal diseases exhibit great variability in clinical expression that contributes to the current inability to predict their rate of progression, severity, and response to therapy, which underscores the need for biomarkers. ${ }^{19}$ A biomarker is defined as a laboratory measurement that reflects the activity of a disease process. Urine or plasma $\mathrm{Gb}_{3}$ concentrations have been used as biomarkers for diagnostic purposes and to follow the effect of specific therapies such as ERT. ${ }^{1,2}$ In addition, the measurement of plasma $\mathrm{Gb}_{3}$ concentration was used as a pharmacodynamic marker to demonstrate the biological activity of agal $\alpha$ in vivo. ${ }^{20}$ For patients with elevated plasma or urine $\mathrm{Gb}_{3}$ concentrations before treatment, ERT resulted in an initial drop in $\mathrm{Gb}_{3}$ concentrations. ${ }^{7,8}$ Lower $\mathrm{Gb}_{3}$ concentrations did not always coincide with clinical improvement.

In this post hoc analysis of prospective RCTs of agalo ERT in Fabry disease, plasma $\mathrm{Gb}_{3}$ concentration was not found to be a significant predictor at month 12 of CFB eGFR (for renal function) or CFB LVMI (for cardiac structure) in the analysis population or any subgroup. Urine $\mathrm{Gb}_{3}$ excretion also failed to predict CFB LVMI at month 12 . However, baseline urine $\mathrm{Gb}_{3}$ excretion did appear to be a predictor of change in renal function, but when analyzed by subgroup, urine $\mathrm{Gb}_{3}$ excretion did not consistently correlate with disease stage, progression, or response to ERT (e.g., baseline urine $\mathrm{Gb}_{3}$ excretion predicted renal function in the placebo $\rightarrow$ agal $\alpha$ and $\mathrm{CKD} 2 / 3$ subgroups but not in the agal $\alpha \rightarrow$ agal $\alpha$ subgroup). CFB of urine $\mathrm{Gb}_{3}$ excretion at month 12 , however, was not found to be a predictor of renal function, suggesting that change in $\mathrm{Gb}_{3}$ concentrations during the course of therapy would not be a good biomarker for response to therapy or Fabry disease progression. Urine protein excretion (baseline and CFB at month 12) was 
Table 4 Potential predictors of renal function: linear regression analyses in subgroup populations

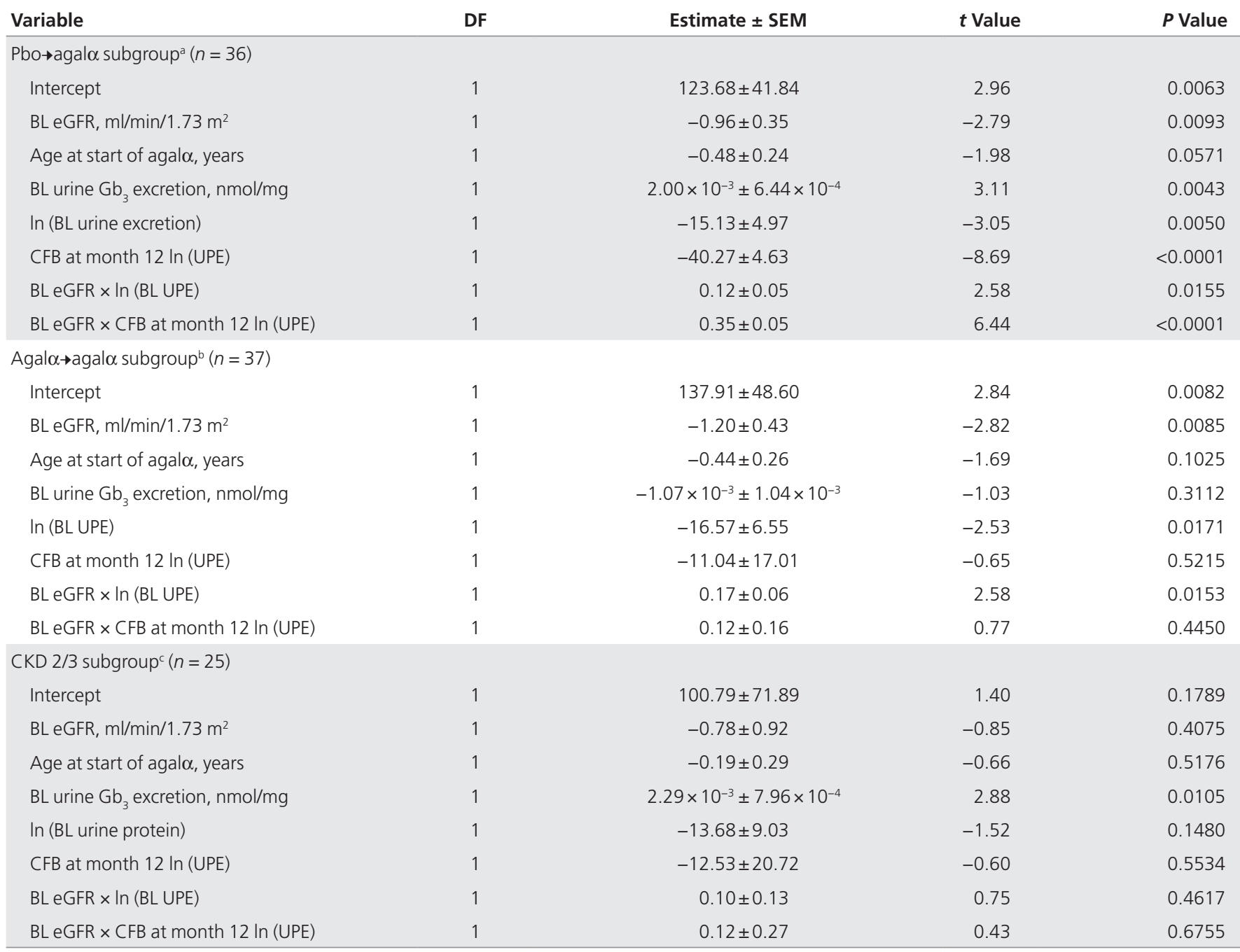

Agalo, agalsidase alfa; BL, baseline; CFB, change from BL; CKD, chronic kidney disease; DF, degrees of freedom; eGFR, estimated glomerular filtration rate; EXT, extension study; $\mathrm{Gb}_{3}$, globotriaosylceramide; Pbo, placebo; $\mathrm{RCT}$, randomized controlled trial; UPE, urine protein excretion.

apbo $\rightarrow$ agal $\alpha$ subgroup: 6 months of placebo (RCT) followed by 12 months of agal $\alpha$ (EXT). ${ }^{b}$ Agal $\alpha \rightarrow$ agal $\alpha$ subgroup: 6 months of agal $\alpha$ (RCT) followed by 6 months of agal $\alpha$ (EXT). ' ${ }^{C}$ KD 2/3 subgroup: stage 2/3 CKD before beginning agal $\alpha$ treatment.

retained in the model as a predictor of renal function in the overall renal analysis population, with statistically significant interaction effects detected with baseline eGFR. In addition, baseline urine protein excretion predicted renal function in the placebo $\rightarrow$ agal $\alpha$ and agal $\alpha \rightarrow$ agal $\alpha$ subgroups, and CFB in urine protein excretion at month 12 was found to be a predictor in the placebo $\rightarrow$ agal $\alpha$ subgroup.

Currently available published evidence does not support the use of plasma or urine $\mathrm{Gb}_{3}$ concentrations as predictive biomarkers for disease progression in patients with Fabry disease. For example, in a cross-sectional analysis in a population of 96 Dutch patients with Fabry disease, concentrations of $\mathrm{Gb}_{3}$ in plasma and urine did not correlate with any clinical symptoms assessed, including renal dysfunction. ${ }^{8}$ The findings of the present analyses are consistent with these studies. Although baseline urine $\mathrm{Gb}_{3}$ excretion was found to be a significant predictor, this may have been of a reflection of baseline Fabry disease severity before agal $\alpha$ treatment initiation; e.g., patients with more severe Fabry disease at baseline may have experienced worse disease outcomes than patients with less progressed Fabry disease before treatment. To assess formally whether plasma and urine $\mathrm{Gb}_{3}$ concentrations would be predictive biomarkers over the longer term for the clinical outcome in the natural history of Fabry disease, a longitudinal study in untreated subjects would be necessary.

The present study has several limitations. Because three trials were analyzed, the pooled patient population was heterogeneous because of differences in inclusion/exclusion criteria. These trials were not designed to evaluate renal function as a primary end point. The primary end point of one trial (TKT010) was changed from neuropathic pain to renal function after 15 months. The original inclusion criteria of this trial, however, were not modified to coincide with this change. In addition, study subjects with Fabry disease were not required 
to have abnormal baseline eGFR and LVMI, which might leave them at lower risk of developing progressive kidney disease or cardiac deterioration during the course of these relatively short-term studies. Although a few significant predictors of renal function were found in the overall analysis population (e.g., baseline eGFR, age at start of agal $\alpha$, baseline urine $\mathrm{Gb}_{3}$ excretion, baseline and CFB at month 12 in urine protein excretion), further research is needed to determine if the lack of statistically significant differences in these same predictors in the subgroups were due to any specific characteristic(s) of these subgroups or were attributable to the patient number being too small to detect an effect. One indication that the patient numbers may have been insufficient in the subgroups is that only the renal analysis population and the placebo $\rightarrow$ agal $\alpha$ subgroup retained CFB at month 12 in urine protein excretion as a statistically significant predictor. Furthermore, a backwardelimination regression model is not a hypothesis-driven model; nevertheless, we consider it the best choice for these exploratory post hoc analyses; however, the follow-up period on ERT was relatively short, so additional analyses may be needed to assess long-term effects. Finally, although some studies support globotriaosylsphingosine as a potentially useful biomarker for monitoring Fabry disease, this evidence has been generated in relatively recent years. ${ }^{6,21,22}$ At the time of the design of the clinical trials included in the current report, globotriaosylsphingosine was not considered a potential surrogate marker of Fabry disease progression or response to treatment and, thus, was not included as a measured end point.

The biological reason why changes in plasma or urine $\mathrm{Gb}_{3}$ concentrations are not useful as biomarkers is not clear. Blood and urine $\mathrm{Gb}_{3}$ concentrations are not mechanistically directly related to the outcome measures used in the current study; urine $\mathrm{Gb}_{3}$ excretion is mostly derived from the renal collecting system, whereas the outcome (eGFR) reflects glomerular function. Likewise, changes in plasma $\mathrm{Gb}_{3}$ do not necessarily represent its turnover in the heart muscle. Because renal function in most patients was not in the rapidly declining stage, the possibility exists that significant reduction in urine or plasma $\mathrm{Gb}_{3}$ concentrations could not be reflected in changes in eGFR over a relatively short period of 12 months. Another possible explanation for our findings is that $\mathrm{Gb}_{3}$ concentrations may not participate in the pathogenic process of renal glomerulopathy or hypertrophic cardiomyopathy of Fabry disease. Therefore, $\mathrm{Gb}_{3}$ may be an example of a biomarker that is not entirely in the causal pathway of the disease process. ${ }^{23}$

In conclusion, the lack of correlation between CFB at month 12 of urine and plasma $\mathrm{Gb}_{3}$ concentrations and renal outcomes suggests that changes in their levels do not predict clinical progression in Fabry disease; however, the discordant baseline urine $\mathrm{Gb}_{3}$ excretion results in the subgroup analyses raises some question as to whether such a relationship exists and requires additional study. Other molecules, for example, globotriaosylsphingosine or reactive oxygen species, may also likely be active participants in the Fabry pathogenic cascade in the kidney and the heart. ${ }^{21,24,25}$

\section{SUPPLEMENTARY MATERIAL}

Supplementary material is linked to the online version of the paper at http://www.nature.com/gim

\section{ACKNOWLEDGMENTS}

These studies and analyses were funded by Shire Human Genetic Therapies (HGT). Medical writing support for this article was provided by Ray Beck of UBC-Envision Group and was funded by Shire HGT.

\section{DISCLOSURE}

R.S. has received honoraria and research support from Shire Human Genetic Therapies (HGT), Amicus Therapeutics, and Genzyme. K.N. has served on the Fabry Outcomes Survey advisory board that is funded by Shire HGT and has received travel and research support from Shire HGT, Genzyme, and Amicus; her center participates in clinical trials funded by Shire HGT and Amicus. A.M. has received research support and speaking honoraria from Shire HGT, Genzyme, and Actelion. J.T.R.C. has received research funding, consultancy fees, and/or speaker fees from Shire HGT, Genzyme, and Actelion. R.D.S. has participated in clinical trials funded by TKT/Shire HGT, Actelion, Genzyme, Protalix/Pfizer, and BioMarin. He has had research support from TKT and has received speaking or consulting fees from TKT/Shire HGT, Amicus, Actelion, Genzyme, Zacharon, and BioMarin. M.B. has received unrestricted grants, honoraria, and travel support from Shire HGT, Genzyme, BioMarin, and Actelion. B.A.B. has received research support from Genzyme and has served on an advisory board for BioMarin. W.R. has received research funding, consultancy fees, and/or speaker fees from Shire HGT, Genzyme, Hyperion, Ucyclyd, and Actelion and has served on advisory boards for Shire HGT. M.W. has received research support from Shire HGT and Genzyme; has received speaking honoraria from Shire HGT, Genzyme, Amicus Therapeutics, and Sumitomo Pharma; and has participated in advisory boards for Shire HGT. M.R. was an employee of Shire HGT from 2006 to 2009; he has served on advisory boards for Alexion, GSK, and Amicus and has received consultancy honoraria from Alexion, Oxyrane, and Shire HGT. P.H. was an employee of Shire HGT from 2008 to 2011. R.M. and N.N are employees of Shire HGT. D.A. was an employee of Shire HGT from 2010 to 2012. D.B. declares no potential competing interests.

\section{REFERENCES}

1. Fuller M, Sharp PC, Rozaklis T, et al. Urinary lipid profiling for the identification of Fabry hemizygotes and heterozygotes. Clin Chem 2005;51:688-694.

2. Auray-Blais C, Cyr D, Mills K, Giguère R, Drouin R. Development of a filter paper method potentially applicable to mass and high-risk urinary screenings for Fabry disease. J Inherit Metab Dis 2007;30:106.

3. Auray-Blais C, Cyr D, Ntwari A, et al. Urinary globotriaosylceramide excretion correlates with the genotype in children and adults with Fabry disease. Mol Genet Metab 2008;93:331-340.

4. Auray-Blais C, Millington DS, Young SP, Clarke JT, Schiffmann R. Proposed highrisk screening protocol for Fabry disease in patients with renal and vascular disease. J Inherit Metab Dis 2009;32:303-308.

5. Young E, Mills K, Morris P, et al. Is globotriaosylceramide a useful biomarker in Fabry disease? Acta Paediatr Supp/ 2005;94:51-4; discussion 37.

6. Togawa T, Kodama T, Suzuki T, et al. Plasma globotriaosylsphingosine as a biomarker of Fabry disease. Mol Genet Metab 2010;100:257-261. 
7. Schiffmann R, Ries M, Timmons M, Flaherty JT, Brady RO. Long-term therapy with agalsidase alfa for Fabry disease: safety and effects on renal function in a home infusion setting. Nephrol Dial Transplant 2006;21:345354.

8. Vedder AC, Linthorst GE, van Breemen MJ, et al. The Dutch Fabry cohort: diversity of clinical manifestations and $\mathrm{Gb}_{3}$ levels. J Inherit Metab Dis 2007:30:68-78.

9. Katz R. Biomarkers and surrogate markers: an FDA perspective. NeuroRx 2004;1:189-195.

10. Center for Drug Evaluation and Research (CDER), Food and Drug Administration, U.S. Department of Health and Human Services. Guidance for Industry - qualification process for drug development tools. http://www. fda. gov/downloads/Drugs/GuidanceComplianceRegulatorylnformation/Guidances/ UCM230597.pdf. Accessed 21 November 2012.

11. West M, Nicholls K, Mehta A, et al. Agalsidase alfa and kidney dysfunction in Fabry disease. J Am Soc Nephrol 2009;20:1132-1139.

12. Schiffmann R, Kopp JB, Austin HA 3rd, et al. Enzyme replacement therapy in Fabry disease: a randomized controlled trial. JAMA 2001;285:2743-2749.

13. Hughes DA, Elliott PM, Shah J, et al. Effects of enzyme replacement therapy on the cardiomyopathy of Anderson-Fabry disease: a randomised, double-blind, placebo-controlled clinical trial of agalsidase alfa. Heart 2008;94:153-158.

14. Levey AS, Stevens LA, Schmid CH, et al.; CKD-EPI (Chronic Kidney Disease Epidemiology Collaboration). A new equation to estimate glomerular filtration rate. Ann Intern Med 2009;150:604-612.

15. K/DOQI. K/DOQI clinical practice guidelines for chronic kidney disease: evaluation, classification, and stratification. Am J Kidney Dis 2002;39:S1S266.

16. Schiffmann R, Murray GJ, Treco D, et al. Infusion of alpha-galactosidase A reduces tissue globotriaosylceramide storage in patients with Fabry disease. Proc Natl Acad Sci USA 2000;97:365-370.
17. Aerts JM, Kallemeijn WW, Wegdam W, et al. Biomarkers in the diagnosis of lysosomal storage disorders: proteins, lipids, and inhibodies. J Inherit Metab Dis 2011;34:605-619.

18. Cox TM. Biomarkers in lysosomal storage diseases. In: Mehta A, Beck M, Sunder-Plassmann G (eds). Fabry Disease: Perspectives From 5 Years of FOS Oxford PharmaGenesis: Oxford, England, 2006.

19. Grabowski GA. Treatment perspectives for the lysosomal storage diseases Expert Opin Emerg Drugs 2008;13:197-211.

20. Ries M, Clarke JT, Whybra C, et al. Enzyme replacement in Fabry disease: pharmacokinetics and pharmacodynamics of agalsidase alpha in children and adolescents. J Clin Pharmacol 2007:47:1222-1230.

21. Aerts JM, Groener JE, Kuiper S, et al. Elevated globotriaosylsphingosine is a hallmark of Fabry disease. Proc Natl Acad Sci USA 2008;105:2812-2817.

22. Rombach SM, Dekker N, Bouwman MG, et al. Plasma globotriaosylsphingosine: diagnostic value and relation to clinical manifestations of Fabry disease. Biochim Biophys Acta 2010;1802:741-748

23. Fleming TR, DeMets DL. Surrogate end points in clinical trials: are we being misled? Ann Intern Med 1996;125:605-613.

24. Auray-Blais $C$, Ntwari $A$, Clarke JT, et al. How well does urinary lyso-Gb function as a biomarker in Fabry disease? Clin Chim Acta 2010;411:1906-1914.

25. Shen JS, Meng XL, Moore DF, et al. Globotriaosylceramide induces oxidative stress and up-regulates cell adhesion molecule expression in Fabry disease endothelial cells. Mol Genet Metab 2008:95:163-168.

(c) This work is licensed under a Creative
License. To view a copy of this license, visit http://
creativecommons.org/licenses/by/3.0/deed.en_US

